\title{
Rapid genetic typing of diarrheagenic Escherichia coli using a two-tube modified molecular beacon based multiplex real-time PCR assay and its clinical application
}

Qingliang Chen ${ }^{1+}$, Xiaolu Shi ${ }^{2 \dagger}$, Yinghui $\mathrm{Li}^{2}$, Yixiang Jiang ${ }^{3}$, Yiman $\mathrm{Lin}^{2}$, Yaqun Qiu ${ }^{2}$, Qingge $\mathrm{Li}^{1,4^{*}}$ and Qinghua Hu $\mathrm{u}^{2,3^{*}}$

\begin{abstract}
Background: Diarrheagenic Escherichia coli (DEC), including Enterotoxigenic E.coli (ETEC), Enteroaggregative E.coli (EAEC), Enteropathogenic E.coli (EPEC), Enterohemolysin E.coli (EHEC) and Enteroinvasive E.coli (EIEC) causes diarrhea or hemolytic uremic syndromes among infants and travelers around the world. A rapid, reliable and repeatable method is urgent for identifying DEC so as to provide the reference for responding to diarrheal disease outbreak and the treatment of the diarrheal patients associated with DEC.

Methods: In this study, specific primers and modified molecular beacon probes of nine specific virulence genes, whose $5^{\prime}$ end were added with homo tail sequence, were designed; and a two-tube modified molecular beacon based multiplex real-time PCR (rtPCR) assay for the identification of five Escherichia coli pathotypes, including ETEC, EAEC, EPEC, EHEC and EIEC was developed and optimized. Totally 102 bacterial strains, including 52 reference bacterial strains and 50 clinical strains were detected to confirm whether the target genes selected were specific. Then detection limits of the assay were tested. Lastly, the assay was applied to the detection of 11860 clinical samples to evaluate the specificity and sensitivity of the developed assay compared with the conventional PCR.

Results: The target genes were $100 \%$ specific as assessed on 102 bacterial strains since no cross-reactions were observed. The detection limits ranged from $88 \mathrm{CFU} / \mathrm{mL}$ (EHEC) to $880 \mathrm{CFU} / \mathrm{mL}$ (EPEC). Compared with the conventional PCR, the specificity and sensitivity of the multiplex rtPCR was 100\% and over $99 \%$, respectively. The coefficient of variation (CV) for each target gene ranged from $0.45 \%$ to $1.53 \% .171$ positive clinical samples were mostly identified as ETEC $(n=111,64.9 \%)$ and EPEC $(n=38,22.2 \%)$, which were the dominating pathotypes of DEC strains.
\end{abstract}

Conclusion: The developed multiplex rtPCR assay for the identification of DEC was high sensitive and specific and could be applied to the rapid identification of DEC in clinical and public health laboratories.

Keywords: Diarrheagenic Escherichia coli, Two-tube multiplex real-time PCR, Modified molecular beacon, Identification, Application

\footnotetext{
*Correspondence: qgli@xmu.edu.cn; huqinghua03@163.com

${ }^{\dagger}$ Equal contributors

${ }^{1}$ Engineering Research Center of Molecular Diagnostics, Ministry of

Education, Department of Biomedical Sciences, School of Life Sciences,

Xiamen University, Xiamen 361005, Fujian Province, China

${ }^{2}$ Shenzhen Major Infectious Disease Control Key Laboratory, Shenzhen

Centre for Disease Control and Prevention, Shenzhen 518055, Guangdong

Province, China

Full list of author information is available at the end of the article
}

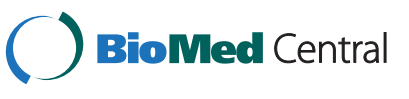

(c) 2014 Chen et al.; licensee BioMed Central Ltd. This is an Open Access article distributed under the terms of the Creative Commons Attribution License (http://creativecommons.org/licenses/by/4.0), which permits unrestricted use, distribution, and reproduction in any medium, provided the original work is properly credited. The Creative Commons Public Domain Dedication waiver (http://creativecommons.org/publicdomain/zero/1.0/) applies to the data made available in this article, unless otherwise stated. 


\section{Background}

Diarrheal disease has been considered as one of the most important public health problem, as well as the significant cause leading to children's death [1] and brings high disease burden to society [2]. Diarrheagenic Escherichia coli (DEC) is the main pathogen associated with diarrheal disease.

Based on the epidemiological, clinical features, specific virulence determinants and other molecular characteristics, DEC has been classified into five main pathotypes as follows: Enterotoxigenic E.coli (ETEC), Enteroaggregative E.coli (EAEC), Enteropathogenic E.coli (EPEC), Enterohemolysin E.coli (EHEC) and Enteroinvasive E.coli (EIEC) [2]. Each pathotype possesses specific virulence genes associated with the disease symptoms. For example, shiga-like toxin I (stx1), shiga-like toxin II (stx2), eaeA and escV genes are virulence genes for EHEC. EaeA encodes intimin that involved in attachment of bacteria to enterocytes, and $e s c V$ is the main component of ring structure on inner membrane. EPEC also contains eaeA and escV, but without shiga-like toxin [3]. For ETEC, it carries the plasmid encoded heat-stable (ST) and/or heat-labile (LT) toxins. ST can be divided into two main groups, stp (also referred as STIa) and sth (also referred as STIb) [4]. Gene $i p a H$, which is related to invasion [5] expressed in EIEC and shigella spp.. The defining feature of EAEC is the distinctive aggregative-adherence pattern, which is mediated by $\operatorname{aggR}$ gene [6].

DEC will mostly result in persistent watery diarrhea, abdominal cramping and diarrhea. EHEC, such as O157: $\mathrm{H} 7$ will cause hemolytic uremic syndrome (HUS) and is life-threatening. DEC is also associated with foodborne disease outbreak or infantile diarrheal disease outbreak. So identification of different pathotypes of DEC will provide the critical information for determining appropriate therapies for patients with suspected E.coli infections and controlling of the outbreak. A variety of assays have been published for identification of DEC. Serotyping and biochemistry has been widely applied in the diagnosis of gastrointestinal pathogens, but they cannot be used for conclusive identification of DEC groups [7]. Molecular assays [8-18], like DNA hybridization [8], loop-mediated isothermal amplification (LAMP) [9], multiplex PCR $[10,11,15,17,18]$, and real-time PCR (rtPCR)-SYBR green melting curve [12-14] have been described for such identification. Among these techniques, multiplex PCR is one of the most useful diagnostic methods, however, this method needs post gel electrophoresis, which is labor-consuming and can easily result in carry-over contamination. Thus it is not suitable for analyzing large numbers of clinical samples in clinical laboratory. Guion et al. [14] creatively used rtPCR SYBR green meltingcurve to detect eight virulence gene of DEC, and the results could be obtained from the peak of melting temperature $\left(\mathrm{T}_{\mathrm{m}}\right)$. However, SYBR green melting-curve is not a stable method, since it's prudent to standardize the assay using control strains in each laboratory.

We have developed a multiplex rtPCR assay based multicolor combinational probe coding (MCPC) for the identification of eight foodborne pathogens [19] and applied to the rapid diagnosis of foodborne disease outbreak successfully. In order to quickly screen DEC from large number of stool samples in laboratory-based surveillance network of diarrheal disease and respond to diarrheal disease outbreak associated with DEC, in this paper, based on the principle of MCPC, we attempted to design modified molecular beacons and primers with homo tail sequences and also add internal amplification control to establish the multiplex real-time PCR assay for the identification of DEC. Considering the different modes of the real-time PCR instruments used in the different hospitals, we established a two-tube multiplex rtPCR assay for detecting nine genes simultaneously with the same thermocycling condition and DEC can be typed within $90 \mathrm{~min}$.

\section{Results}

\section{Detection limits and reproducibility of multiplex rtPCR}

The detection limits of this method for bacterial pure culture ranged from $88 \mathrm{CFU} / \mathrm{mL}$ (EHEC) to $880 \mathrm{CFU} / \mathrm{mL}$ (EPEC). Amplification efficiencies for bacterial culture ranged from $92.2 \%$ (EIEC) to $102.8 \%$ (EHEC) (Figure 1 and Table 1).

The $C_{T}$ values from three replicate experiments for each ten-fold dilution $\left(10^{6} \mathrm{cfu} / \mathrm{ml}-10^{3} \mathrm{cfu} / \mathrm{ml}\right)$ differed within one cycle and the coefficient of variation (CV) for each target gene ranged from $0.45 \%$ to $1.53 \%$, which indicated that the assay was reproducible (Table 2).

\section{Specificity of target genes for multiplex rtPCR}

All 102 bacterial strains were correctly identified and no cross-reaction or false positives were observed, demonstrating that the selected target genes were specific for their respective pathotypes (Additional file 1: Table S1).

\section{Application of multiplex rtPCR for the identification of suspected DEC samples}

Of the 11860 stool samples, 5100 samples were putatively E.coli positive on MacConkey agar and biochemical test. Among 5100 suspected DEC samples, 171 were DEC positive, including 38 ETEC, 111 EPEC, four EHEC, 10 EAEC and eight EIEC identified by multiplex rtPCR assay, whereas 150 were DEC positive including 29 ETEC, 99 EPEC, four EHEC, 10 EAEC and eight EIEC were identified by the conventional PCR.

When compared with the conventional PCR, the sensitivity of the multiplex rtPCR assay was 100\% (95\% CIs $39.76-100 \%)$ for all five pathotypes detected, and the 

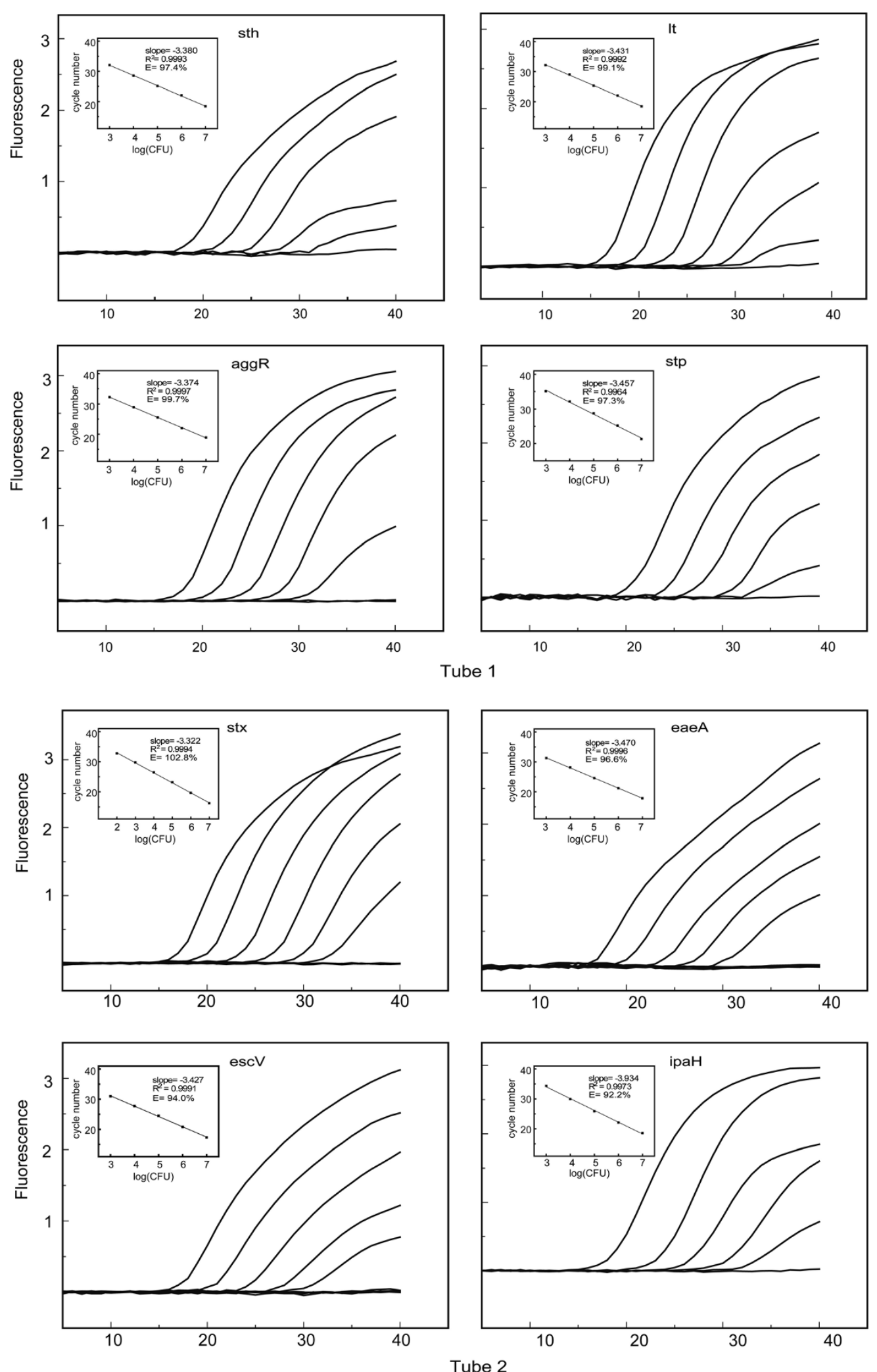

Figure 1 Detection limits of five main pathotypes of diarrheagenic Escherichia coli. Ten-fold serial dilutions of bacterial cultures of five diarrheagenic Escherichia coli pathotypes were prepared to assess the detection limits. 
Table 1 Detection limits and amplification efficiency of multiplex rtPCR assay

\begin{tabular}{|c|c|c|c|c|c|c|c|}
\hline Tube & Pathotypes & Virulence gene & Equation & $\mathrm{R}^{2}$ & Efficiency & Liner range (CFU/mL) & Detection limits (CFU/mL) \\
\hline \multirow[t]{4}{*}{ Tube 1} & ETEC & stp & $y=-3.345 x+45.797$ & 0.9964 & $97.3 \%$ & $3.0 \times 10^{2} \sim 3.0 \times 10^{6}$ & $3.0 \times 10^{2}$ \\
\hline & & sth & $y=-3.386 x+42.136$ & 0.9993 & $97.4 \%$ & $3.0 \times 10^{2} \sim 3.0 \times 10^{6}$ & $3.0 \times 10^{2}$ \\
\hline & & It & $y=-3.343 x+42.537$ & 0.9992 & $99.1 \%$ & $3.0 \times 10^{2} \sim 3.0 \times 10^{7}$ & $3.0 \times 10^{2}$ \\
\hline & EAEC & $\operatorname{agg} R$ & $y=-3.371 x+42.398$ & 0.9997 & $99.7 \%$ & $2.0 \times 10^{2} \sim 2.0 \times 10^{6}$ & $2.0 \times 10^{2}$ \\
\hline \multirow[t]{4}{*}{ Tube 2} & EHEC & $s t \times 1 / 2$ & $y=-3.257 x+39.610$ & 0.9994 & $102.8 \%$ & $8.8 \times 10^{1} \sim 8.8 \times 10^{6}$ & $8.8 \times 10^{1}$ \\
\hline & EPEC & eaeA & $y=-3.405 x+41.464$ & 0.9996 & $96.6 \%$ & $8.8 \times 10^{2} \sim 8.8 \times 10^{6}$ & $8.8 \times 10^{2}$ \\
\hline & & escV & $y=-3.475 x+41.401$ & 0.9991 & $94.0 \%$ & $8.8 \times 10^{2} \sim 8.8 \times 10^{6}$ & $8.8 \times 10^{2}$ \\
\hline & EIEC & $i p a H$ & $y=-3.523 x+45.832$ & 0.9973 & $92.2 \%$ & $6.8 \times 10^{2} \sim 6.8 \times 10^{6}$ & $6.8 \times 10^{2}$ \\
\hline
\end{tabular}

specificity for ETEC, EPEC, EHEC, EAEC, and EIEC was 99.92\%, 99.90\%, 100\%, 100\% and 100\%, respectively (95\% CIs 99.82-100\%). Kappa value for ETEC, EPEC, EHEC, EAEC, and EIEC was 0.87, 0.94, 1.00, 1.00 and 1.00, respectively (Table 3).

\section{Distribution of DEC pathotypes among 171 DEC positive clinical samples}

Among 171 DEC positive samples, the positive rate of ETEC and EPEC were 64.9\% (111/171) and 22.2\% (38/ $171)$, respectively. And the positive rate of EAEC, EIEC, EHEC were 5.8\% (10/171), 4.7\% (8/171) and 2.3\% (4/171), respectively. Besides, the data showed that adults were the main infected group, which accounted for $82.5 \%$ $(141 / 171)$. The infection rate of children (age $\leq 6$ year) was only $12.9 \%$ (22/171). Obvious monthly peaks of DEC infections were found during the warmer months from June to September; approximately $73.7 \%(126 / 171)$ of diarrheal patients could be attributed to DEC infections during this period (Figure 2).

\section{Discussion}

In present study, two-tube multiplex rtPCR was successfully established for identifying five pathotypes of DEC using homo tail primers and modified molecular beacons simultaneously. The detection limits ranged from $88 \mathrm{CFU} /$ $\mathrm{mL}$ to $880 \mathrm{CFU} / \mathrm{mL}$. Compared with the conventional PCR assay, detection data of 11860 clinical samples showed that two-tube multiplex rtPCR possessed $100 \%$ sensitivity and above $99 \%$ specificity. After optimizing cycling condition of the assay, five categorized DEC were detected at the same time, leading to a great reduction of cost and time. And the time required from DNA extraction to final result was less than 90 min. Therefore the assay was a high sensitive, specific and rapid method.

Traditionally, high throughput rtPCR was restricted by the wavelength of fluorescence channel and primer dimer formation, which may result in simultaneous detection of two or three genes in one reaction. The follows were used as means to overcome these limitations: First, part of the stem structure of probes was designed to participate in hybridization of template to increase the binding affinity and reduce the background of fluorescence signal greatly. Second, to reduce dimer formation, homo-tag assisted non-dimer (HAND) method $[20,21]$ was put into use to ensure the stability and sensitivity of multiplex rtPCR. Also, the additional tag sequence increased the $T_{m}$ value of primer, making the optimization of the same cycling temperature for twotube assay easier. The above methods are able to enlarge

Table 2 The reproducibility of the two-tube multiplex real-time PCR assay

\begin{tabular}{|c|c|c|c|c|c|c|c|c|}
\hline \multirow[t]{2}{*}{ genes } & \multicolumn{2}{|l|}{$10^{6} \mathrm{cfu} / \mathrm{ml}$} & \multicolumn{2}{|l|}{$10^{5} \mathrm{cfu} / \mathrm{ml}$} & \multicolumn{2}{|l|}{$10^{4} \mathrm{cfu} / \mathrm{ml}$} & \multicolumn{2}{|l|}{$10^{3} \mathrm{cfu} / \mathrm{ml}$} \\
\hline & Mean Ct \& SD & $\overline{C V}(\%)$ & Mean Ct \& SD & $\overline{C V}(\%)$ & Mean Ct \& SD & $\overline{C V}(\%)$ & Mean Ct \& SD & $\overline{C V}(\%)$ \\
\hline eaeA & $21.22 \pm 0.18$ & 0.85 & $24.58 \pm 0.22$ & 0.90 & $28.14 \pm 0.31$ & 1.10 & $31.26 \pm 0.26$ & 0.83 \\
\hline escV & $20.8 \pm 0.13$ & 0.63 & $24.49 \pm 0.11$ & 0.45 & $27.73 \pm 0.25$ & 0.90 & $30.99 \pm 0.32$ & 1.03 \\
\hline ipaH & $22.11 \pm 0.18$ & 0.81 & $25.85 \pm 0.31$ & 1.12 & $29.93 \pm 0.22$ & 0.74 & $34.34 \pm 0.458$ & 1.31 \\
\hline Stx $1 / 2$ & $19.64 \pm 0.18$ & 0.92 & $23.16 \pm 0.09$ & 0.39 & $26.44 \pm 0.21$ & 0.79 & $29.74 \pm 0.2$ & 0.67 \\
\hline $\operatorname{aggR}$ & $22.02 \pm 0.17$ & 0.77 & $25.52 \pm 0.31$ & 1.21 & $28.95 \pm 0.39$ & 1.35 & $32.3 \pm 0.28$ & 0.87 \\
\hline It & $22.06 \pm 0.14$ & 0.63 & $25.29 \pm 0.31$ & 1.23 & $28.99 \pm 0.22$ & 0.76 & $32.13 \pm 0.49$ & 1.53 \\
\hline sth & $22.04 \pm 0.0 .13$ & 0.58 & $25.11 \pm 0.31$ & 1.23 & $28.54 \pm 0.4$ & 1.40 & $32.07 \pm 0.25$ & 0.78 \\
\hline stp & $25.2 \pm 0.09$ & 0.36 & $28.76 \pm 0.29$ & 1.01 & $32.19 \pm 0.23$ & 0.71 & $35.1 \pm 0.21$ & 0.60 \\
\hline
\end{tabular}


Table 3 Sensitivity and specificity tested using multiplex real-time PCR and conventional PCR in 11860 clinical samples

\begin{tabular}{|c|c|c|c|c|c|c|c|c|c|}
\hline \multirow[t]{2}{*}{ DEC group } & \multirow{2}{*}{$\begin{array}{l}\text { Multiplex } \\
\text { real-time } \\
\text { PCR }\end{array}$} & \multicolumn{2}{|c|}{ Standard PCR } & \multirow[t]{2}{*}{ Total } & \multirow[t]{2}{*}{ Sensitivity } & \multirow[t]{2}{*}{$95 \% \mathrm{Cl}$} & \multirow[t]{2}{*}{ Specificity } & \multirow[t]{2}{*}{$95 \% \mathrm{Cl}$} & \multirow[t]{2}{*}{ Kappo } \\
\hline & & $t^{1}$ & $-^{2}$ & & & & & & \\
\hline \multirow[t]{3}{*}{$\overline{E T E C}$} & + & 29 (a) & 9 (b) & 38 & $100 \%$ & $88.06 \sim 100.00$ & $99.92 \%$ & $99.86 \sim 99.97$ & 0.87 \\
\hline & - & 0 (c) & $11822(d)$ & 11822 & & & & & \\
\hline & Total & 29 & 11831 & 11860 & & & & & \\
\hline \multirow[t]{3}{*}{ EPEC } & + & 99 & 12 & 111 & $100 \%$ & $96.34 \sim 100.00$ & $99.90 \%$ & $99.82 \sim 99.95$ & 0.94 \\
\hline & - & 0 & 11749 & 11749 & & & & & \\
\hline & Total & 99 & 11761 & 11860 & & & & & \\
\hline \multirow[t]{3}{*}{ EHEC } & + & 4 & 0 & 4 & $100 \%$ & $39.76 \sim 100.00$ & $100 \%$ & $99.97 \sim 100.00$ & 1.00 \\
\hline & - & 0 & 11856 & 11856 & & & & & \\
\hline & Total & 4 & 11856 & 11860 & & & & & \\
\hline \multirow[t]{3}{*}{ EAEC } & + & 10 & 0 & 10 & $100 \%$ & $69.15 \sim 100.00$ & $100 \%$ & $99.97 \sim 100.00$ & 1.00 \\
\hline & - & 0 & 11850 & 11850 & & & & & \\
\hline & Total & 10 & 11850 & 11860 & & & & & \\
\hline \multirow[t]{3}{*}{ EIEC Positive } & + & 8 & 0 & 8 & $100 \%$ & $63.06 \sim 100.00$ & $100 \%$ & $99.97 \sim 100.00$ & 1.00 \\
\hline & - & 0 & 11852 & 11852 & & & & & \\
\hline & Total 171 & 8150 & 11852 & 11860 & & & & & \\
\hline
\end{tabular}

$+^{1}:$ Positive, $-^{2}$ : Negative.

the signal-to-noise ratio, and enhance the efficiency of amplification. Comparing to gel electrophoresis and SYBR green melting curve, the use of fluorescence probes would avoid reopening the reaction tubes, thus greatly reduce the risk for carry-over contamination and false-positive and will also avoid errors from band discrimination when observed by naked eyes.

Comparing to the conventional PCR for DEC identification according to diagnostic criteria for infectious diarrhea (WS 271-2007, China) and other literature data, our assay covers more genes, such as esc $V$, stp and sth. Since eaeA had a lot of single nucleotide polymorphisms, esc $V$ was also included for the identification of

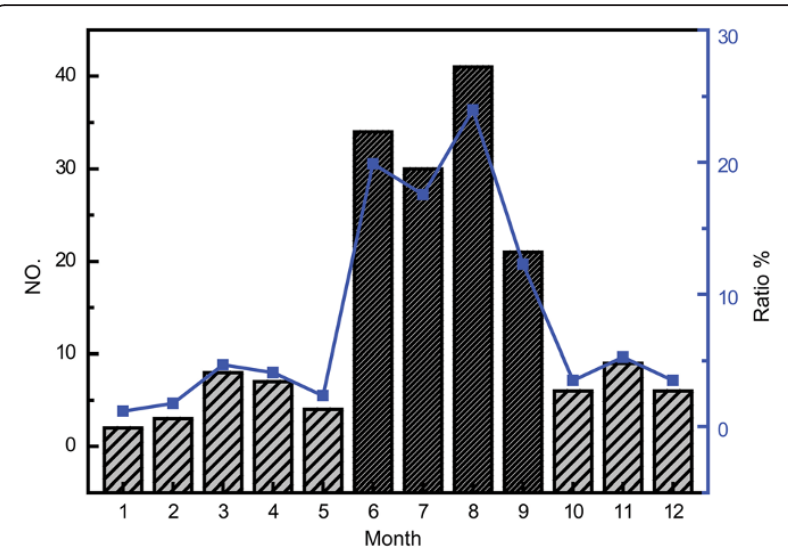

Figure 2 Monthly distribution (in bar) and ratio (point) of diarrheagenic Escherichia coli clinical isolates in 2011 to 2012.
EPEC. The twelve extra positive samples were identified when esc $V$ was added. In addition, only using st gene without differentiation of $s t p$ and $s t h$ will lead to nine ETEC missing. Statistical analysis revealed that there was no difference among positive result of EAEC, EHEC and EIEC using multiplex rtPCR and the conventional PCR. All these suggested that more genes were preferred to improve the sensitivity of DEC identification method.

In order to indicate false-negative of multiplex rtPCR, an internal amplification control (IAC) was added to the multiplex rtPCR assay. We used the sequence of the human adenovirus (accession No. AY601634) as an internal amplification control to guarantee that proper reagents, such as magnesium, Taq polymerase and $1 \times$ PCR buffer were suitable for PCR amplification.

Considering that clinical DEC strains should be isolated for the further antibiotics susceptibility test, we adapted the multiplex rtPCR to the suspected colonies for the identification of DEC, not directly to the fresh stool samples. Based on the research data of Barletta et al. [12], we used five-colony pool analysis from one clinical sample for typing DEC.

Epidemiological information of out-patients was collected, including patients'age and symptoms (data not shown). Among 171 positive cases, adults were the main infected population instead of children. This may be due to the population structure of Shenzhen, in which young workers constitute a big percentage of population. For DEC cases, ETEC was the predominant type, accounting for $64.9 \%$ of all cases. This was corresponded to a new 
systematic review [22]. During summer, diarrhea cases caused by DEC increased rapidly (Figure 2), suggesting a high risk of infection in summer and an essential measure to take to promote health care.

In conclusion, a rapid and effective two-tube multiplex rtPCR for the identification of all DEC pathotypes was established. This method was time (90 $\mathrm{min})$ and resources saving (including biochemical reagents, disposable medical supplies) and could be used in clinical and public health laboratories.

\section{Materials and methods}

\section{Reference bacterial strains and clinical strains}

A total of 111 bacterial strains, comprising 24 species, including 61 reference bacterial strains from American Type Culture Collection (ATCC) or National Center for Medical Culture Collections of China (CMCC) and 50 clinical isolates, were collected as specific or nonspecific targets (Additional file 1: Table S1). Nine specific reference strains were selected for establishing multiplex rtPCR (four types of shigella spp:: CMCC 51265, CMCC 51376, ATCC 12022, CMCC 51081; ETEC: ATCC 35401; EAEC: ATCC 33780; EIEC: ATCC 43893; EHEC: ATCC 35150; and EPEC: ATCC 43887). All strains were confirmed by diagnostic criteria for infectious diarrhea published by Chinese Centre for Disease Control and Prevention (WS 271-2007) using the conventional PCR (Additional file 1: Table S2).

\section{Bacterial culture and DNA extraction}

All positive reference bacterial strains were cultured on appropriate agar plates to isolate bacterial colonies. After overnight incubation at $37^{\circ} \mathrm{C}$, single colonies of each culture were carefully picked and added into $200 \mu \mathrm{l}$ sterile purified water. After shaking thoroughly and heating for five minute, slurry was centrifuged at $12000 \mathrm{rpm}$ for one minute, and $5 \mu \mathrm{l}$ supernatant was collected for PCR reaction.

Clinical stool samples were streaked on MacConkey agar plates. After overnight incubation at $37^{\circ} \mathrm{C}$, a pool of five suspicious colonies was picked for DNA extraction as follows. A pool of five suspicious colonies was added to $200 \mu \mathrm{l}$ sterile purified water and shaked thoroughly following heating lyses for five minute. After centrifuging at $12000 \mathrm{rpm}$ for one minute, $5 \mu \mathrm{l}$ supernatant was assimilated for PCR reaction.

\section{Primers and probes}

Specific virulence genes were chosen to detect specific pathogens: ipaH for EIEC, stp/sth/lt for ETEC, eaeA/ esc $V$ for EPEC, stx1/stx2 for EHEC, aggR for EAEC. The sequence of the human adenovirus (accession No. AY601634) was selected as an internal amplification control(IAC) for the multiplex rtPCR assay [23]. All the sequences of genes published in the Genbank were downloaded and aligned by Clustal X 2.0. Conserved regions were picked up for designing primers and probes after merging into GenBank. Based on the articles published online [24], molecular beacon probes were designed with the help of DNA folding form website (http://mfold.rit.albany.edu/?q=mfold/DNA-Folding-Form). All the 5 'end of primers were added with a homo tail sequence to alleviate the forming of primer dimer. Molecular beacons were modified and designed. All primers and probes were listed in Table 4.

\section{Performance of multiplex rtPCR}

The homo tail was added to $5^{\prime}$ end of primer sequences so that proper annealing temperature will fall into broad range in each individual PCR reaction. Five classes of DEC were divided into two tubes. For tube-one, stp/sth/lt for ETEC, $a g g R$ for EAEC and IAC were included, while ipaH for EIEC, eaeA/esc $V$ for EPEC, stx1/stx2 for EHEC and IAC were included in tube-two. Multiplex rtPCR reactions were performed on Bio-Rad CFX 96 Real-Time PCR System (Bio-Rad, Hercules, CA). Firstly, individual real-time PCR cycling condition and proper reagent concentration were optimized. Secondly, two-tube system reagents, including primers, probes, magnesium and Taq polymerase are mixed and optimized separately. The optimal assay was as follows: tube-one, $1 \times$ PCR buffer, $3 \mathrm{mM} \mathrm{MgCl}_{2}, 2 \mu \mathrm{l}$ of deoxynucleoside triphosphate $(2.5 \mathrm{mM})$, and 1 unit Taq polymerase, $0.12 \mu \mathrm{M}$ to $0.3 \mu \mathrm{M}$ specific primers and probes (Table 4) and $5 \mu \mathrm{l}$ of DNA template; tube-two, $1 \times$ PCR buffer, $2.5 \mathrm{mM} \mathrm{MgCl} 2,2 \mu \mathrm{l}$ of deoxynucleoside triphosphate $(2.5 \mathrm{mM})$, and 1 unit Taq polymerase, $0.2 \mu \mathrm{M}$ to $0.4 \mu \mathrm{M}$ specific primers and probes (Table 4 ) and $5 \mu \mathrm{l}$ of DNA template. The amplification cycles consisted of a first stage of denature at $95^{\circ} \mathrm{C}$ for $3 \mathrm{~min}$; a second stage of $5 \mathrm{cy}$ cles: $95^{\circ} \mathrm{C}$ for $5 \mathrm{~s}, 58^{\circ} \mathrm{C}$ for $15 \mathrm{~s}, 72^{\circ} \mathrm{C}$ for $15 \mathrm{~s}$; a third stage of 40 cycles: $95^{\circ} \mathrm{C}$ for $5 \mathrm{~s}, 55^{\circ} \mathrm{C}$ for $15 \mathrm{~s}, 72^{\circ} \mathrm{C}$ for $15 \mathrm{~s}$. Carboxy fluorescein (FAM), Hexachloro fluorescein (HEX), Carboxy-X-rhodamine (ROX), Quasar 705 and indodicarbocyanine5 (Cy5) fluorescence were collected and recorded at the end of annealing step during the third stage.

\section{Detection limits and reproducibility of multiplex rtPCR}

Five ATCC reference strains (ETEC ATCC 35401, EAEC ATCC 33780, EIEC ATCC 43893, EHEC ATCC 35150 and EPEC ATCC 43887) were used for the analysis of detection limits. The target strains were cultured in Luria broth and the concentration of the bacteria was adjusted to $10^{6}$ or $10^{7}$ colony forming units $(\mathrm{CFU}) / \mathrm{mL}$ using a turbidity meter, then $1 \mathrm{~mL}$ enrichment broth was used as the dilution, meanwhile an aliquot of each dilution was quantified via plate counting. The DNA template of serial 1:10 dilutions was extracted by heating lyses method mentioned above and was used for the detection limits analysis and four tenfold serial dilutions $\left(10^{6}-10^{3} \mathrm{cfu} / \mu \mathrm{L}\right)$ were prepared for the analysis of the reproducibility in three replicate experiments. 
Table 4 The primers and probes used for multiplex real-time PCR assay in this study ${ }^{a}$ F/R/P: forward and reverse primer, ${ }^{\text {b }}$ concentration

\begin{tabular}{|c|c|c|c|c|c|}
\hline Tube & Group & Target genes & Primers and Probes $^{\mathrm{a}}$ & Sequence(5'-3') & Conc $^{\mathrm{b}}(\mu \mathrm{M})$ \\
\hline \multirow[t]{12}{*}{ Tube 1} & $\mathrm{ETEC}^{1}$ & stp & stp-F & tag-AAAAGCGAGTGTACCTCGACA & 0.18 \\
\hline & & & $s t p-R$ & tag-CAGTTGACTGACTAAAAGAGGGG & 0.3 \\
\hline & & & stp-P & HEX-CGCGTCTCAAATATCCGTGAAACAACATGACGCG-Dabcyl & 0.2 \\
\hline & & sth & sth-F & tag-GTGGTCCTGAAAGCATGAATAG & 0.12 \\
\hline & & & sth-R & tag-CAACAAAGCAACAGGTACATACG & 0.12 \\
\hline & & & sth-P & FAM-CGCGGTGAATTGTGTTGTAATCCTGCTTGTACCGCG-Dabcyl & 0.2 \\
\hline & & It & $\mid t-F$ & tag-ACAGGAGGTTTCTGCGTTAG & 0.18 \\
\hline & & & It $-\mathrm{R}$ & tag-GGTGGGAAACCTGCTAATCT & 0.18 \\
\hline & & & $\mid t-P$ & ROX-CGCCGGTATTACAGAAATCTGAATATAGCTCCGGCG-Dabcyl & 0.2 \\
\hline & EAEC ${ }^{1}$ & $\operatorname{aggR}$ & $\operatorname{aggR-F}$ & tag-TGCAAAAGAAGAAATCAACAGT & 0.18 \\
\hline & & & $\operatorname{agg} R-R$ & tag-CAGAATCGTCAGCATCAGCTAC & 0.3 \\
\hline & & & aggR-P & CY5-CGGACAAAAGTAGATGCTTGCAGTTGTCCG-Dabcyl & 0.2 \\
\hline \multirow[t]{15}{*}{ Tube 2} & $\mathrm{EIEC}^{2}$ & ipaH & ipaH-F3 & tag-GAAAACCCTCCTGGTCCATC & 0.32 \\
\hline & & & ipaH-R3 & tag-GTCTGGAAGGCCAGGTAGACTT & 0.32 \\
\hline & & & ipaH-P & FAM-CCCGGCTGGAGGACATTGCCCGGG-Dabcyl & 0.2 \\
\hline & EPEC\& & escl & escV-F & tag-GGCTCTCTTCTTCTITATGGCTG & 0.4 \\
\hline & $\mathrm{EHEC}^{2}$ & & esc $V-R$ & tag-GGGAAAGAAGTTAGTTCAAGAGGAT & 0.4 \\
\hline & & & escV-P & HEX-CCCGCGCAACAGTTGTGGTGGATATCATTATCGCGGG-Dabcyl & 0.2 \\
\hline & & eaeA & eaeA-F & tag-GTAACCAGGCTTCGTCACA & 0.32 \\
\hline & & & eaeA-R & tag-AAGGAAAAAACGCTGACCCG & 0.32 \\
\hline & & & eaeA-P & CY5-CCCAGTGGTAATAACTTTGACGGTAGTTCACTGGG-Dabcyl & 0.2 \\
\hline & $\mathrm{EHEC}^{2}$ & $s t \times 1$ & $s t \times 1-\mathrm{F}$ & tag-ASAGCGGTTACATTGTCTGGT & 0.24 \\
\hline & & & $s t \times 1-\mathrm{R}$ & tag-CTGCGTCAGTGAGGTTCCA & 0.24 \\
\hline & & & $s t \times 1-\mathrm{P}$ & ROX-CCGCGTACGGGGATGCAGATAAATCGCGG-Dabcyl & 0.2 \\
\hline & & $s t \times 2$ & $s t \times 2-\mathrm{F}$ & tag-CATGACAACGGACAGCAGTTA & 0.24 \\
\hline & & & $s t \times 2-\mathrm{R}$ & tag-TCTGGTCATTGTATTACCACTGAA & 0.24 \\
\hline & & & $s t \times 2-\mathrm{P}$ & ROX-CCGCCACTCACTGGTTTCATCATATCTGGCGG-Dabcyl & 0.2 \\
\hline \multirow[t]{4}{*}{ Tube $1 \& 2$} & $\mid A C^{1,2}$ & & IAC-F & GGCGCGCCTAACACATCT & 0.1 \\
\hline & & & IAC-R & TGGAAGCAATGCCAAATGTGTA & 0.1 \\
\hline & & & ICA-P & CGGTGGTTACAACGGGAGAAGACAATGCCACCG & 0.2 \\
\hline & & & $\operatorname{tag}$ & GCAAGCCCTCACGTAGCGAA & 2.4 \\
\hline
\end{tabular}

${ }^{1}:$ tube $1,{ }^{2}$ : tube2.

IAC: Internal amplification control.

\section{Specificity of multiplex rtPCR assay}

To confirm whether the target genes selected were specific for each pathotype of DEC, 102 well-characterized strains were tested by multiplex rtPCR.

\section{Application of multiplex rtPCR for the identification of suspected clinical samples}

The multiplex rtPCR was applied to detecting clinical samples to determine assay sensitivity and specificity with direct comparison with conventional PCR. All clinical samples were collected based on the inclusion criteria of World
Health Organization (WHO) case definition for diarrhea. Diarrheal disease was described as the follows: having three or more loose or liquid stools per day, or as having more stools than normal person. The stool samples of totally 11860 out-patients were collected for DEC detection from 11 sentinel hospitals during 2011 to 2012 in Shenzhen, Guangdong province, China.

All specimens were streaked onto MacConkey agar and a minimum of five typical suspected E.coli colonies were picked and identified by biochemical test and the conventional PCR according to the diagnostic criteria for 
infectious diarrhea (WS 271-2007, China). Then DNA of a pool of five typical suspected colonies was extracted and performed for both two-tube multiplex rtPCR and conventional PCR simultaneously. For conventional PCR, the reaction volume and amplification condition were as the follows: $5 \mu \mathrm{l}$ of DNA template was applied to PCR reaction at the final volume of $50 \mu \mathrm{l}$; the PCR reaction consisted of $10 \mathrm{mM}$ Tris- $\mathrm{HCl}$ (pH 8.3), $50 \mathrm{mM} \mathrm{KCl,} 0.1 \%$ Triton X-100, $1.5 \mathrm{mM} \mathrm{MgCl}_{2}$, $2.5 \mathrm{U}$ Taq, $0.2 \mathrm{mMdNTP}$, and $0.125-0.5 \mu \mathrm{M}$ primer pairs. The cycling condition of PCR contained $95^{\circ} \mathrm{C}$ for $10 \mathrm{~min}$; and 35 cycles of $94^{\circ} \mathrm{C}$ for $1 \mathrm{~min}, 52^{\circ} \mathrm{C}$ for $1 \mathrm{~min}, 72^{\circ} \mathrm{C}$ for $1 \mathrm{~min}$; then $72^{\circ} \mathrm{C}$ for $10 \mathrm{~min}$. PCR products were analyzed with $1.5 \%$ agarose gel electrophoresis. The primer sequences were attached in Additional file 1: Table S2.

\section{Statistical analysis}

The results of the multiplex $\mathrm{rtPCR}$ assay were compared with conventional PCR using a $2 \times 2$ table to estimate indices of sensitivity and specificity. Corresponding 95\% confidence intervals (CIs) were calculated by using approximate normal distribution and exact interval methods and Kappa value was calculated.

\section{Additional file}

Additional file 1: Table S1. Reference and clinical strains used for multiplex real-time PCR assay.

\section{Competing interests}

The authors declare that they have no competing interests.

\section{Authors' contributions}

QC designed primers and modified molecular beacons of target genes for EIEC and EPEC and carried out multiplex real-time PCR assay. XS designed primers and modified molecular beacons of target genes for EAEC and EHEC. $Y L$ participated in traditional PCR assay. YJ designed primers and modified molecular beacons of target genes for ETEC. YL and YQ participated in bacteria culture and DEC biochemical identification. QL participated in the design of the study. QH conceived of the study, and participated in its design and coordination and revised the manuscript. All authors read and approved the final manuscript.

\section{Acknowledgements}

This work was supported by National Natural Science Foundation of China (No. 81071433 to Q.H), Guangdong Provincial Natural Science Foundation (No. 8151802003000006 to Q.H), and Shenzhen Public Service Platform of Pathogenic Microorganisms Repository and supported by Basic Research Project of Shenzhen (No. JC201104220329A to Q.H and Q.Li).

\section{Author details}

${ }^{1}$ Engineering Research Center of Molecular Diagnostics, Ministry of Education, Department of Biomedical Sciences, School of Life Sciences, Xiamen University, Xiamen 361005, Fujian Province, China. ${ }^{2}$ Shenzhen Major Infectious Disease Control Key Laboratory, Shenzhen Centre for Disease Control and Prevention, Shenzhen 518055, Guangdong Province, China. ${ }^{3}$ School of Life Sciences, Shenzhen University, Shenzhen 518000, Guangdong, China. ${ }^{4}$ Shenzhen Research Institute of Xiamen University, Shenzhen 518057, Guangdong, China.
Received: 25 March 2014 Accepted: 5 July 2014

Published: 15 July 2014

\section{References}

1. Gostin LO: Meeting basic survival needs of the world's least healthy people: toward a framework convention on global health. Geo $U 2007$, 96:331.

2. Brandal LT, Lindstedt BA, Aas L, Stavnes TL, Lassen J, Kapperud G: Octaplex PCR and fluorescence-based capillary electrophoresis for identification of human diarrheagenic Escherichia coli and Shigella spp. J Microbiol Methods 2007, 68:331-341.

3. Nataro JP, Kaper JB: Diarrheagenic escherichia coli. Clin Microbiol Rev 1998 11:142-201.

4. Fleckenstein JM, Hardwidge PR, Munson GP, Rasko DA, Sommerfelt H, Steinsland H: Molecular mechanisms of enterotoxigenic Escherichia coli infection. Microbes Infect 2010, 12:89-98.

5. van den Beld MJ, Reubsaet FA: Differentiation between Shigella, enteroinvasive Escherichia coli (EIEC) and noninvasive Escherichia coli. Eur J Clin Microbiol Infect Dis 2012, 31:899-904.

6. Kaur P, Chakraborti A, Asea A: Enteroaggregative Escherichia coli: an emerging enteric food borne pathogen. Interdiscip Perspect Infect Dis 2010, 2010:1-10.

7. Persson S, Olsen K, Scheutz F, Krogfelt K, Gerner-Smidt P: A method for fast and simple detection of major diarrhoeagenic Escherichia coli in the routine diagnostic laboratory. Clin Microbiol Infect 2007, 13:516-524.

8. Scaletsky IC, Fabbricotti SH, Aranda KR, Morais MB, Fagundes-Neto U: Comparison of DNA hybridization and PCR assays for detection of putative pathogenic enteroadherent Escherichia coli. J Clin Microbiol 2002, 40:1254-1258.

9. Yokoyama $\mathrm{E}$, Uchimura M, Ito K: Detection of enteroaggregative Escherichia coli by loop-mediated isothermal amplification. J Food Prot 2010, 73:1064-1072

10. Botkin DJ, Galli L, Sankarapani V, Soler M, Rivas M, Torres AG: Development of a multiplex PCR assay for detection of Shiga toxin-producing Escherichia coli, enterohemorrhagic E. Coli, and enteropathogenic E. Coli strains. Frontiers Cellular Infect Microbiol 2012, 2:1-10.

11. Taniuchi M, Walters CC, Gratz J, Maro A, Kumburu H, Serichantalergs O, Sethabutr O, Bodhidatta L, Kibiki G, Toney DM: Development of a multiplex polymerase chain reaction assay for diarrheagenic Escherichia coli and Shigella spp. and its evaluation on colonies, culture broths, and stool. Diagn Microbiol Infect Dis 2012, 73:121-128.

12. Barletta F, Ochoa TJ, Ecker L, Gil Al, Lanata CF, Cleary TG: Validation of five-colony pool analysis using multiplex real-time PCR for detection of diarrheagenic Escherichia coli. J Clin Microbiol 2009, 47:1915-1917.

13. Bischoff $C$, Luthy J, Altwegg M, Baggi F: Rapid detection of diarrheagenic E. coli by real-time PCR. J Microbiol Methods 2005, 61:335-341.

14. Guion CE, Ochoa TJ, Walker CM, Barletta F, Cleary TG: Detection of diarrheagenic Escherichia coli by use of melting-curve analysis and real-time multiplex PCR. J Clin Microbiol 2008, 46:1752-1757.

15. Antikainen J, Tarkka E, Haukka K, Siitonen A, Vaara M, Kirveskari J: New 16-plex PCR method for rapid detection of diarrheagenic Escherichia coli directly from stool samples. Eur J Clin Microbiol Infect Dis 2009, 28:899-908.

16. Vidal M, Kruger E, Duran C, Lagos R, Levine M, Prado V, Toro C, Vidal R: Single multiplex PCR assay to identify simultaneously the six categories of diarrheagenic Escherichia coli associated with enteric infections. J Clin Microbiol 2005, 43:5362-5365.

17. Fujioka M, Kasai K, Miura T, Sato T, Otomo Y: Rapid diagnostic method for the detection of diarrheagenic Escherichia coli by multiplex PCR. Jpn J Infect Dis 2009, 62:476-480.

18. Chandra M, Cheng P, Rondeau G, Porwollik S, McClelland M: A single step multiplex $\mathrm{PCR}$ for identification of six diarrheagenic $E$. coli pathotypes and Salmonella. Int J Med Microbiol 2013, 303:210-216.

19. Hu Q, Lv D, Shi X, Jiang Y, Lin Y, Li Y, Qiu Y, He L, Zhang R, LiA Q: Modified molecular beacons-based multiplex real-time PCR assay for simultaneous detection of eight foodborne pathogens in a single reaction and its application. Foodborne Pathogens 2014, 11(3):207-214

20. Brownie J, Shawcross S, Theaker J, Whitcombe D, Ferrie R, Newton C, Little S: The elimination of primer-dimer accumulation in PCR. Nucleic Acids Res 1997, 25:3235-3241.

21. Goyer C, Dandie CE: Quantification of microorganisms targeting conserved genes in complex environmental samples using quantitative polymerase chain reaction. Quantitative Real-Time Pcr Applied Microbiol 2012, 87-106. 
22. Walker CLF, Sack D, Black RE: Etiology of diarrhea in older children, adolescents and adults: a systematic review. PLoS Negl Trop Dis 2010, 4:e768.

23. Suo B, He Y, Tu S-I, Shi X: A multiplex real-time polymerase chain reaction for simultaneous detection of Salmonella spp., Escherichia coli 0157, and Listeria monocytogenes in meat products. Foodborne Pathogens Dis 2010, 7:619-628.

24. Tyagi S, Kramer FR: Molecular beacons: probes that fluoresce upon hybridization. Nat Biotechnol 1996, 14:303-308.

doi:10.1186/s12941-014-0030-8

Cite this article as: Chen et al:: Rapid genetic typing of diarrheagenic

Escherichia coli using a two-tube modified molecular beacon based

multiplex real-time PCR assay and its clinical application. Annals of Clinical

Microbiology and Antimicrobials 2014 13:30.

\section{Submit your next manuscript to BioMed Central and take full advantage of:}

- Convenient online submission

- Thorough peer review

- No space constraints or color figure charges

- Immediate publication on acceptance

- Inclusion in PubMed, CAS, Scopus and Google Scholar

- Research which is freely available for redistribution 